\title{
Modelling of a Novel Configuration for Single-belt Caster: The Influence of Empirical Parameters on the Solidification Profile
}

\author{
Pedro Gutemberg Q. NETTO and Roderick I. L. GUTHRIE ${ }^{11}$ \\ Formerly at the McGill Metals Processing Centre, now at Universidade Federal Fluminense, Volta Redonda-Brazil, Av. dos \\ Trabalhadores, 420-Vila Santa Ceília. Volta Redonda-RJ, 27260-740, Brazil. E-mail: pedro@metal.eeimvr.uff.br \\ 1) McGill Metals Processing Centre, McGill University, M.H. Wong Building, 3610 University Street, Montreal, Qc-H3A 2B2 \\ Canada. E-mail: rod@mmpc.mcgill.ca
}

(Received on March 9, 1999; accepted in final form on January 11, 2000)

\begin{abstract}
A mathematical model was developed to simulate fluid flow/heat transfer phenomena in a proposed configuration for a single-belt caster. The main goal of the research was to evaluate the flow modifications yielded by the insertion of a flow modifier in the computational domain. This paper deals in particular with the influence of some empirical parameters in the model predictions. The most important inputs for the mathematical model were found to be the interfacial heat transfer coefficient $h$ and the morphology constant $C$. These two parameters, in addition to the numerical treatment for turbulence, had a remarkable influence on the model's outputs. A very high value of $C$ generates strong dampening of velocities within the interdendritic, mushy zone. This leads to predictions of premature solid shell growth within the extended metal delivery zone, leading to too thick a solid shell and too thin a mushy zone at the end of the computational domain. As for the instantaneous heat transfer coefficient, $h$, an attempt was made to predict its transient characteristics using an experimental simulator. This equipment mimics metal deposition on a substrate moving at the same velocity as an industrial belt $(\sim 0.5-1 \mathrm{~m} / \mathrm{s})$. Comparisons of predictions made on the basis of different schemes for the variation in $h$ are reported. The importance of an accurate prediction for the way $h$ varies, in terms of the belt's required cooling length, is stressed.
\end{abstract}

KEY WORDS: single-belt strip casting; heat flux measurements; flow through porous media.

\section{Introduction}

From the beginning of the 1980's, much research has been directed to develop so called "near net shape casting" on a commercial scale. Various processes, such as roll processes and belt processes are being tested in many different countries. ${ }^{1,2)}$ For steel castings, the major developments have been the thin slab casters, developed in the last ten years.

Recently, $\mathrm{Cramb}^{3)}$ reviewed trends in the strip casting of steel. In Fig. 1, he compares the potential productivities of casters with those achieved. As seen, the Nippon Yakin Twin Roll has a productivity similar to the Thin Slab process, but these are considerably lower than conventional continuous casting processes. The only current process under investigation that has the potential to match the productivity of conventional casting machines is the single-belt casting process being investigated at Clausthal and MEFOS. Cramb also gives a comparison of the cast speeds achieved by the various technologies (Fig. 2), where it is shown that the final strip thicknesses in the roll processes $(1-7 \mathrm{~mm})$ is significantly less than the strand thicknesses in the MEFOS project $(\sim 10 \mathrm{~mm})$. The reason, according to Spitzer and Schwerdtfeger, ${ }^{4)}$ for the philosophy of the single-belt caster is that hot rolling is necessary for most steel grades, prior to cold rolling, to obtain strip properties comparable to those obtained in conventional continuous casting processes. As such, in this process, belt speeds and strand thicknesses are in the order of $1 \mathrm{~m} / \mathrm{s}$ and $10 \mathrm{~mm}$, respectively. This allows hot rolling with 50 to $80 \%$ reduction, and eases the requirements on surface quality and dimensional uniformity. ${ }^{4}$ )

In the specific case of single-belt processes, one of the most important boundary conditions is the interfacial heat transfer between the solidifying strip and the moving substrate. The determination of interfacial heat fluxes and heat transfer coefficients has been the subject of exhaustive studies in the literature. However, most of these measurements have monitored heat transfer rates between a static mold (foundry and conventional continuous casting processes) and the cast metal. Also, casting speeds have been much lower (usually zero) and strand thicknesses and solidification times considerably higher, than those pertaining to near-net-shape casting processes. These process variables are of primary importance for heat flux characteristics, as shown by Wang and Matthys. ${ }^{5)}$

The main purpose of this paper is to further assess the potential merits of the single-belt horizontal caster, and to explore some of the difficulties related to the quality of strips produced by these single-belt strip casting processes. 


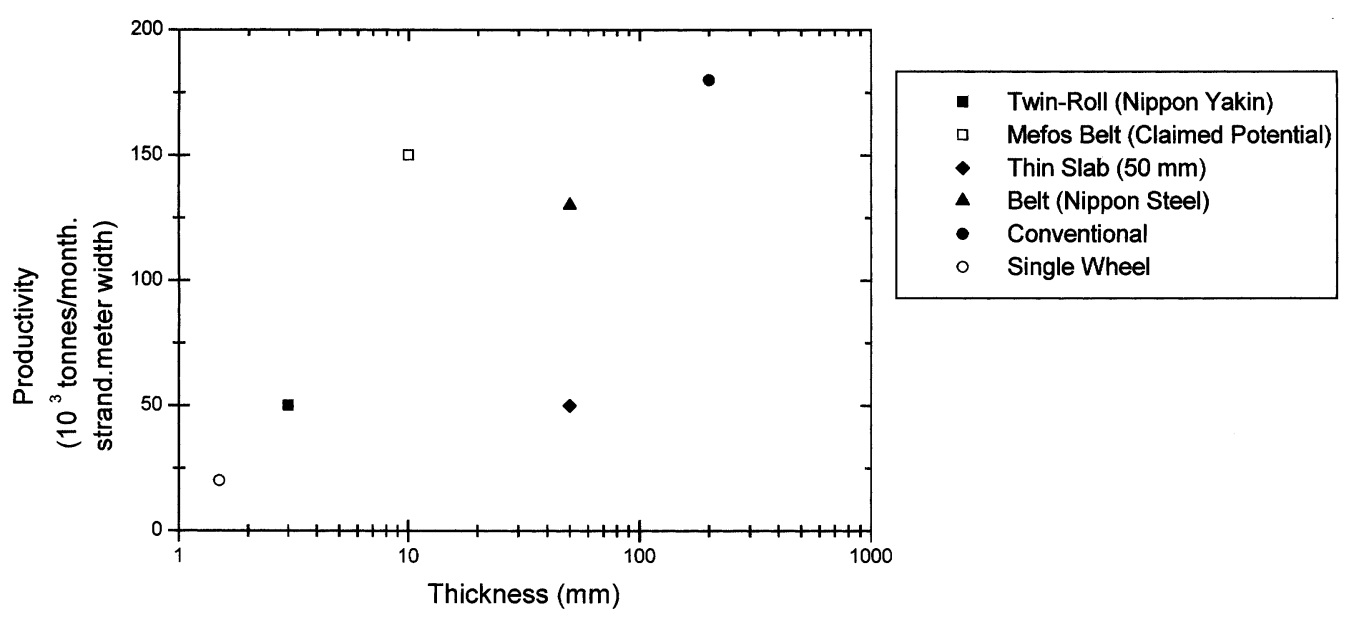

Fig. 1. Achieved and potential productivies of casters. ${ }^{1)}$

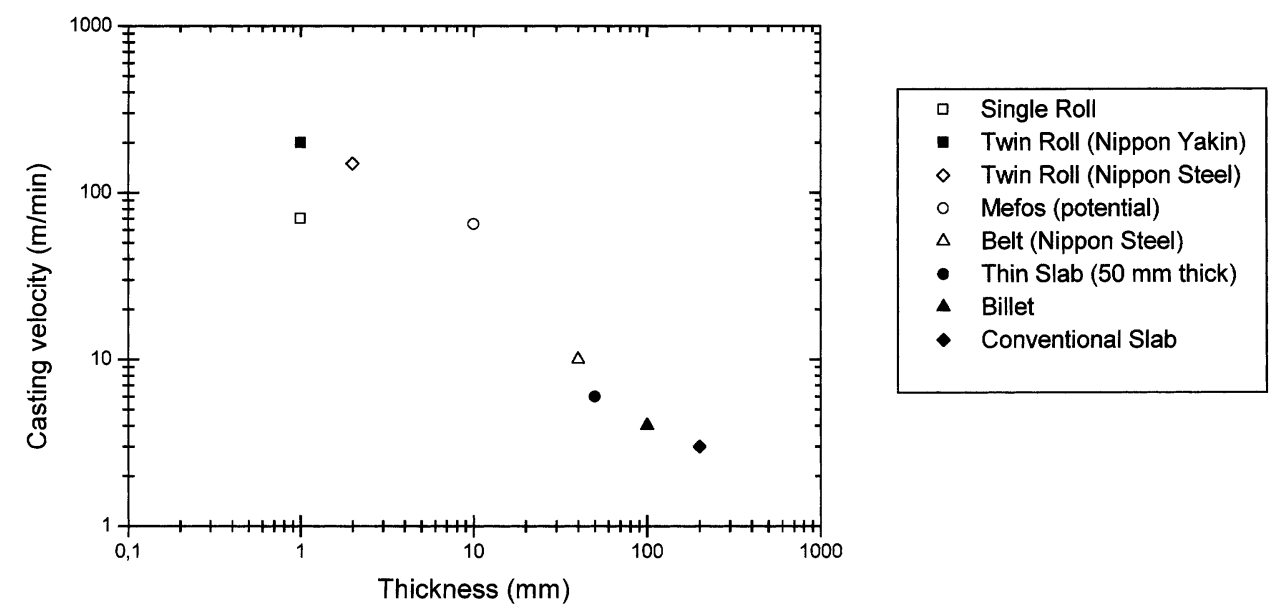

Fig. 2. Achieved casting speeds of various technologies. ${ }^{1)}$

A metal delivery system is proposed and a comprehensive mathematical model, including heat transfer, fluid flow and stress studies is used as a tool to perform this task. This model includes three-dimensional flows with fully coupled momentum and heat transfer. Turbulence effects as deduced from the standard $\kappa-\varepsilon$ equations, solidification, as well as flow through porous media are modeled.

Along with the mathematical model, an experimental apparatus was developed for the present research, which aimed at reproducing, as closely as possible, the type of metal-substrate contact occurring in a single-belt caster. Casting thin strips at high speeds is studied by carrying out parametric analyses, for the most important variables affecting the horizontal casting process. Similarly, an inverse heat conduction model was used to evaluate the interfacial heat fluxes and heat transfer coefficients likely to be encountered in full scale systems.

This paper deals with the effect of some of the most important inputs of the mathematical model on its predictions. Turbulence modelling, in addition to the scheme for the evolution of the interfacial heat transfer coefficient $h$ and also the value of the morphology constant $C$ were found to be the most critical parameters for the mathematical model in representing more accurately the fluid flow/heat transfer phenomena in the proposed delivery system. The impor- tance of turbulence modelling in this configuration is the subject of a separate paper); the present work focuses on the study of $h$ and $C$. An attempt to determine the evolution of $h$ in the experimental apparatus will also be reported.

\section{Development of the Mathematical Model}

\subsection{General Assumptions}

The mathematical model obeyed some predetermined design criteria, with respect to caster productivity and geometrical aspects. Conditions were also superimposed, such as the reduction in the likelihood of a hydraulic jump at the nozzle exit. For that, the kinetic energy of the flow in the exit gap $\left(m v^{2} / 2\right)$ was matched to the potential energy of the inlet $(m g h)$. Figure 3 provides a schematic of the computational domain (symmetry axis).

The conservation of mass, momentum and energy for steady-state flows can be represented by the following partial differential equation, its general form being given by Patankar ${ }^{7)}$ :

$$
\frac{\partial}{\partial x_{i}}\left(\rho u_{i} \phi\right)=\frac{\partial}{\partial x_{i}}\left(\Gamma_{\text {eff }} \frac{\partial \phi}{\partial x_{i}}\right)+S_{\phi}
$$

The most important assumptions adopted in the model 


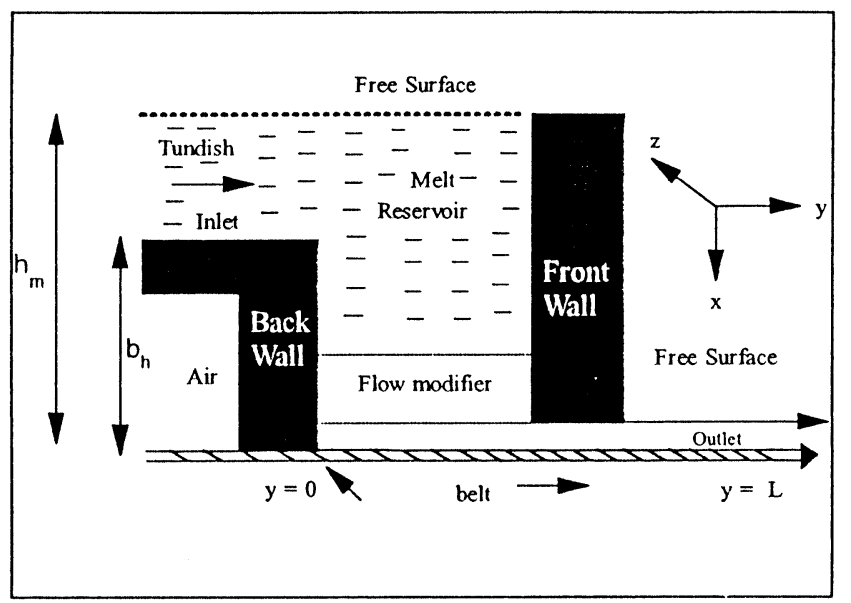

Fig. 3. Schematic of the proposed configuration for the delivery system.

are listed below:

i) The turbulent fluid flow and heat transfer are fully coupled;

ii) The phenomena are essentially three-dimensional and steady-state is assumed;

iii) The metal flow is considered incompressible and the fluid Newtonian;

iv) Columnar dendritic solidification is assumed;

v) A low-Reynolds number $\kappa-\varepsilon$ model was used to account for turbulence within the flow;

vi) The Darcy-Brinkman equation was solved for the porous flow control region;

vii) Thermophysical properties in the liquid, mushy and solid zones were considered constant;

viii) Latent heat of solidification is released between the liquidus and solidus temperature in a uniform manner;

ix) Natural convection effects are not included in the model, since inertial effects dominate;

x) The metal is assumed to be laid down on the belt in an iso-kinetic fashion, i.e., no-slip condition at solidus temperature.

xi) Surface tension effects are not considered in the present analysis;

\subsection{Solidification}

In order to take advantage of the benefits of the fixed grid techniques applied to solidification problems, the continuum model advocated by Bennon and Incropera ${ }^{8)}$ was used in this work.

As for the flow in the mushy zone, it was assumed that the mushy zone behaved as a porous medium. Darcy's law usually applies for columnar dendritic structure and the Carman-Kozeny equation, coming from an extension of Darcy's law, was employed. According to CarmanKozeny's equation, the permeability $\kappa_{\mathrm{p}}$ is related to the liquid fraction $f_{\mathrm{L}}$ according to:

$$
\kappa_{\mathrm{p}}=\frac{f_{\mathrm{L}}^{3}}{C\left(1-f_{\mathrm{L}}^{2}\right)}
$$

The most significant effect of these permeability source terms will be that of making the horizontal $v$-velocity (di- rection of movement) of the solidifying shell approach the belt velocity, at the completion of solidification. For the two other directions, the velocity component will approach zero.

The constant $C$ (Darcy coefficient) defines the morphology and effectively controls the degree of convection field into the mushy zone. ${ }^{9)}$ Its value should be big enough to force the velocity in the solid region to equal the belt speed in the direction of the movement and to zero in the two other directions. Although it is known ${ }^{10)}$ that this coefficient needs to be determined according to the material's microstructure of solidification (dendritic, planar, etc.), values in the range of $10^{5}-1.6 \times 10^{7}$ have been reported in the literature. Considering that cooling rates are expected to be very high in the single-belt process, a value of $1 \times 10^{7}$ was chosen as a standard. Nevertheless, since one of the objectives of these simulations is to determine the importance of $C$ in the morphology of the mushy zone, values of $1 \times 10^{5}$ and $1 \times 10^{9}$ were chosen as low/high limits, respectively.

\subsection{Turbulence}

In this work, a low-Reynolds number model was used to account for turbulence near the walls (front, back and sidewalls). Amongst the low-Re number $\kappa-\varepsilon$ models described in the literature, the model proposed by Launder and Sharma ${ }^{11)}$ was employed in this work. This model is easy to implement in solidification problems, ${ }^{12)}$ and has often been used for metallurgical applications. ${ }^{12-14)}$ In this model, the turbulent viscosity, $\mu_{t}$, is given by:

$$
\mu_{\mathrm{t}}=C_{\mu} f_{\mu} \rho \frac{\kappa^{2}}{\varepsilon}
$$

where $\kappa$ is the turbulent kinetic energy, $\varepsilon$ is the rate of dissipation of $\kappa$ and $C_{\mu}$ is a constant. The function $f_{\mu}$ is proportional to the turbulent Reynolds number $R_{\mathrm{T}}$ :

$$
f_{\mu}=\exp \left[\frac{-3.4}{\left(1+\frac{R_{\mathrm{T}}}{50}\right)^{2}}\right],
$$

where:

$$
R_{\mathrm{T}}=\frac{\rho \kappa^{2}}{\mu \varepsilon}
$$

In the mushy zone, a mechanism proposed by Shyy ${ }^{10)}$ was adopted to damp turbulence:

$$
f_{\mu}=\sqrt{f_{\mathrm{L}}} \cdot f_{\mu^{\prime}}
$$

where $f_{\mathrm{L}}$ is the liquid fraction and $f_{\mu^{\prime}}$ is the original value of $f_{\mu}$.

Modelling turbulence proved to be critical for this particular configuration. Evidence for that is given in an article by Netto and Guthrie. ${ }^{6}$ The presence of a solid body within the computational domain and the sudden contraction experienced by the liquid metal, constitute serious limitations to the applicability of the $a d-h o c$ viscosity approach.

\subsection{Porous Medium}

One of the most important objectives of this mathematical model is to simulate the modifications that result from the incorporation of a flow modifier in the proposed metal delivery system. In this regard, the first important point is to 
determine the nature of the flow.

Therefore, the three flow resistances were initially taken into consideration:

i) The bulk damping resistance due to the porous structure (Darcy's law);

ii) The resistance due to inertial effects (Forchheimer's extension);

iii) The viscous resistance due to the boundary walls (Brinkman's extension).

The first results showed, however, that the Forchheimer term did not cause any appreciable change in the flow results, while increasing the computational complexity. Therefore, for the sake of simplicity and computational savings, this term was not employed for the parametric studies. Indeed, as McDonald et al. ${ }^{15)}$ had concluded, for Re less than 300 , the flow is laminar inside the porous medium and thus the inertial terms should not be significant. In the specific problem, Re, defined on the basis of pore diameter, ranged typically from 40 to 100 .

- Turbulence Modeling for Flow in Porous Media

As discussed in the literature review, there is no tested nor validated model for turbulence within a porous medium. Therefore, the basic approach followed in this work was to apply the $\kappa-\varepsilon$ equations to the whole domain, including the porous medium. Since the flow was found to be essentially laminar within the porous medium region, the solution of the $\kappa-\varepsilon$ equations in this region was basically unnecessary.

\subsection{Boundary Conditions and Thermophysical Prop- erties}

Usually, the boundary conditions originate from in-plant measurements of the relevant variables, such as temperature and flow velocity. In addition, correlations and data existent in the literature are used with reasonable accuracy. In the present case, since the model is supposed to simulate a new design of a hypothetical caster, such information is not available. Therefore, the relevant thermal boundary conditions were approximated according to data published in the literature. Although the primary objective of the model was to study the influence of the most important process variables and the changes in the fluid flow and heat transfer resulting from the inclusion of a porous medium in the proposed delivery system, the influence of empirical parameters, such as the morphology constant $C$ and the interfacial heat transfer coefficient $h$ is of great importance, as well. Therefore, a study was conducted on the influence of such terms in the outputs of the mathematical model.

The following standard boundary conditions were adopted for the turbulent fluid flow:

* No slip on the solid walls. At the moving belt, the velocity in the direction of the movement, $v$, will be equal to the belt velocity and the two others plus $\kappa-\varepsilon$ will be zero;

* At the free surfaces of the melt (both within the reservoir and the exiting strip), the gradients of all variables with respect to $x$ will be zero and the velocity perpendicular to the free surface will also be zero;

* At the symmetry plane (direction $z$ ), the gradient of all variables with respect to $z$ will be zero as well as the corresponding perpendicular velocity;
Table 1. Thermophysical properties of the low-carbon steel and porous media.

\begin{tabular}{ll}
\hline \multicolumn{1}{c}{ Low Carbon Steel } & $\begin{array}{c}\text { Porous media - 92\% } \mathbf{A l 2 O}_{3} \text { foam } \\
\text { filters 45 ppi }\end{array}$ \\
\hline $\begin{array}{ll}\text { Density, } \rho=7020 \mathrm{~kg} / \mathrm{m}^{3} \\
\text { Thermal conductivity, } \mathbf{k}=32.6 \mathrm{~W} / \mathrm{m} . \mathrm{K}\end{array}$ & $\begin{array}{l}\text { Pore size, } \mathbf{D}=420 \mu \mathrm{m} \\
\text { Overall porosity, } \epsilon_{\mathbf{p}}=0.84\end{array}$ \\
Specific heat, $\mathbf{C}_{\mathbf{p}}=680 \mathrm{~J} / \mathrm{kg} . \mathrm{K}$ & Thermal conductivity, $\mathbf{k}_{\mathrm{p}}=1.4 \mathrm{~W} / \mathrm{m} . \mathrm{K}$ \\
\cline { 2 - 2 } Heat of fusion, $\mathbf{L}_{\mathrm{f}}=270 \mathrm{KJ} / \mathrm{kg}$ & Porous media Zirconia ZTA 10 ppi \\
\cline { 2 - 2 } $\begin{array}{l}\text { Solidus temperature, } \mathbf{T}_{\mathbf{s}}=1492^{\circ} \mathrm{C} \\
\text { Liquidus temperature, } \mathbf{T}_{\mathbf{l}}=1535^{\circ} \mathrm{C}\end{array}$ & Pore size, $\mathbf{D}=1443 \mu \mathrm{m}$ \\
Molecular viscosity, $\boldsymbol{\mu}=0.0068 \mathrm{~kg} / \mathrm{m} . \mathrm{s}$ & Overall porosity, $\epsilon_{\mathbf{p}}=0.87$ \\
Emissivity of liquid steel, $\epsilon_{1}=0.28$ & \\
\hline
\end{tabular}

* At the outlet ( $40 \mathrm{~cm}$ from the back wall), fully-developed flow was considered. In addition, the outflow is numerically related to the inlet and the exit gap, in order to accelerate convergence;

* At the inlet, the velocity $v$ is specified and linked to the outflow. Velocities $u$ and $w$ are set to zero. The inlet conditions for the turbulence variables were taken from the literature, ${ }^{16)}$ for extended nozzles:

$$
\kappa_{\mathrm{it}}=0.005 v_{\mathrm{it}}^{2} ; \quad \varepsilon_{\mathrm{it}}=0.09 \frac{\kappa_{\mathrm{it}}^{1.5}}{0.03\left(h_{\mathrm{m}}-b_{\mathrm{w}}\right)}
$$

where $v_{\text {it }}$ is the inlet velocity and $\left(h_{\mathrm{m}}-b_{\mathrm{w}}\right)$ represents the height of the inlet stream.

The energy boundary conditions were, in general:

* The metal loses no heat to the back and lateral walls;

* An initial superheat was specified for the inlet flow;

* The metal on the free surfaces of the reservoir and exiting gap lose heat to the environment by radiation and convection;

* Heat is exchanged by conduction with the side and the bottom of the front wall. A uniform heat flux condition was applied to both sites, equal to $50 \mathrm{~kW} / \mathrm{m}^{2}$;

* The metal mainly loses heat by convective exchange with the substrate moving belt). Since one of the goals of this paper is to evaluate the effect of changes in the scheme for evolution of $h$ in the solidification results, a number of schemes were simulated:

1) $h$ was considered constant and equal to 5000 $\mathrm{W} / \mathrm{m}^{2} \cdot \mathrm{K}$

2) A stepwise variation of $h$ was considered: 10000 $\mathrm{W} / \mathrm{m}^{2} \cdot \mathrm{K}$ in the reservoir, $5000 \mathrm{~W} / \mathrm{m}^{2} \cdot \mathrm{K}$ from the exit nozzle to the end of the front wall and finally $3000 \mathrm{~W} / \mathrm{m}^{2} \cdot \mathrm{K}$ from that point on;

3) Transient evolution of $h$, according to experiments carried out in a strip casting simulator constructed at McGill University ${ }^{17)}$;

* On the outlet, a fully-developed profile was considered for the enthalpy.

The thermophysical properties of the low-carbon steel and porous media are presented in Table $\mathbf{I}$.

\subsection{Numerical Model}

The fully-coupled, nonlinear, partial differential equations (conservation of mass and energy and momentum equations) cannot be solved analytically. Therefore, a control-volume finite difference scheme based on the SIMPLE algorithm (Semi-Implicit Method for Pressure Linked 
Table 2. Standard conditions for the simulations of low-carbon steel strip casting.

\begin{tabular}{clc}
\hline Symbol & \multicolumn{1}{c}{ Description } & Standard Value \\
\hline $\mathbf{d}_{\mathbf{g}}$ & Exit gap, strip thickness & $10 \mathrm{~mm}$ \\
$\mathbf{h}_{\mathbf{m}}$ & Metal head & $7 \mathrm{~cm} ; 14 \mathrm{~cm}$ (filter) \\
$\mathbf{I}$ & Reservoir length & $10 \mathrm{~cm}$ \\
$\mathbf{L}_{\mathbf{d}}$ & Computational domain length & $40 \mathrm{~cm}$ \\
$\mathbf{b}_{\mathbf{w}}$ & Back wall height & $4 \mathrm{~cm}$ \\
$\mathbf{d}_{\mathbf{p}}$ & Filter thickness & $2.5 \mathrm{~cm}$ \\
$\mathbf{n z}$ & Front wall thickness & $2.25 \mathrm{~cm}$ \\
$\mathbf{W}$ & Width of the strip & $45 \mathrm{~cm}$ \\
$\mathbf{v}_{\mathbf{b}}$ & Belt velocity & $1 \mathrm{~m} / \mathrm{s}$ \\
$\mathbf{S}_{\mathbf{h}}$ & Initial superheat & $10^{\circ} \mathrm{C}$ \\
$\mathbf{m}^{\prime}$ & Inlet flowrate & $114 \mathrm{ton} / \mathrm{hr}$ \\
\hline
\end{tabular}

Equations) was employed. The conservation equations were then discretized into algebraic equations and solved with the in-house METFLO code.

Convergence was achieved when the summation of the individual residuals of the conservation equations was smaller than $0.5 \%$, for each variable. The residuals were calculated based on the inlet quantities. Grid dependency was investigated for the standard cases, with and without the flow modifier. For the case without the flow modifier, a $72 \times 56 \times 23$ grid was chosen, after calculations showed that finer grids would yield variations in typical results that were not worth the extra computational time. Table 2 gives the standard conditions for the simulations of low-carbon steel strip casting.

- Factors Affecting Accuracy of the Model

In the study of near-net-shape casting processes, very little information is available concerning the morphology of the mushy zone. This difficulty is not actually a privilege of near-net-shape processes, since not much is known for the conventional continuous casting machines, although these processes have been around for some fourty years.

Besides the lack of information on the morphology of the mushy zone, more accurate values for the overall heat transfer coefficient between the metal and the cooling belt are still in need. Usually, a constant averaged value for $h$ is assigned for the whole length of the cooling belt, which is clearly an idealization.

The purpose of this study is to identify and to investigate the main factors affecting the accuracy of the mathematical model presented herein. To achieve that, the constant $C$, which defines the morphology of the mushy zone, will be varied in the next section, and different profiles for the overall heat transfer coefficient will also be tried.

\section{Effect of the Morphology Constant $C$}

As reported in Ref. 17), Minakawa's ${ }^{18)}$ equation was taken as a first reference to calculate the morphology constant. For the SDAS values reported by Spitzer and Schwerdtfeger, ${ }^{4)}$ for horizontal single-belt casting of stainless steel and from the values found in our experimental apparatus, an average SDAS of $15 \mu \mathrm{m}$ was adopted in this work. This translates, according to Minakawa's equation, to a value of approximately $1 \times 10^{6}$ for $C$. Nevertheless, as al-

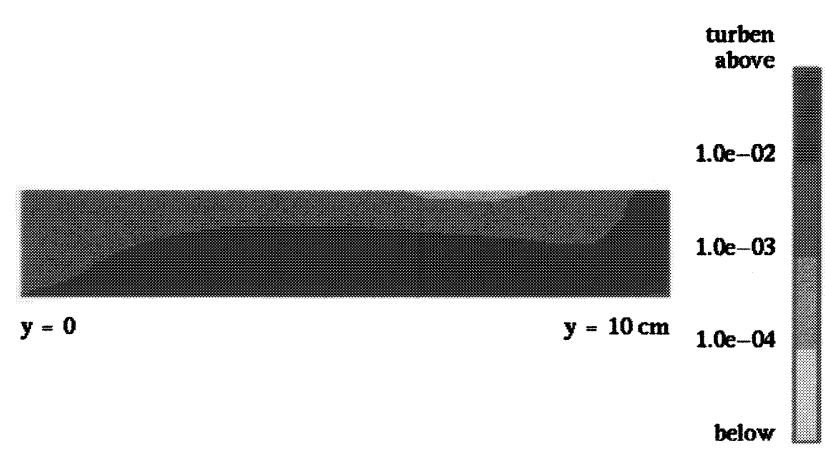

Fig. 4. Turbulent kinetic energy at the symmetry axis of the strip inside the reservoir - $C=1 \times 10^{5}$.

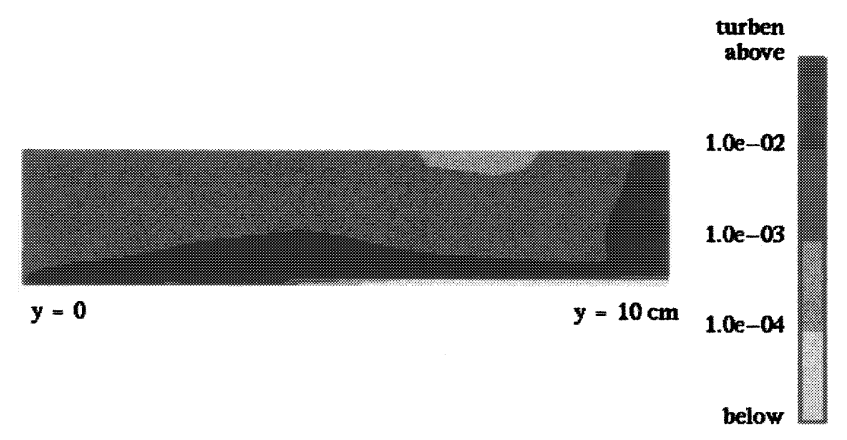

Fig. 5. Turbulent kinetic energy at the symmetry axis of the strip inside the reservoir $-C=1 \times 10^{9}$.

ready mentioned, a value of $1 \times 10^{7}$ was employed in this work, which is closer to the value chosen by Jefferies ${ }^{19)}$ for her two-dimensional model $\left(1.6 \times 10^{7}\right)$.

Thus, taking this value as the standard, the constant was varied by two orders of magnitude, up and down. As such, values of $10^{5}$ and $10^{9}$ were used for $C$, while all other conditions remained unchanged.

i) Fluid Flow Results

No significative changes were noticed on the fluid flow patterns, for the different values of $C$. This is due to the absence of any mushy zone in the reservoir, for the standard conditions, except for the region immediately over the cooling belt.

The turbulent kinetic energy profiles, however, show important changes in the region of the strip inside the reservoir. In Fig. 4, representing a lower value of $C$, considerably more turbulence is seen in the region of the strip. This is a result of a less dampened velocity in the mushy zone, due to a lower Darcy term. On the other hand, for a higher value of $C$ (Fig. 5), the velocity dampening is very intense in the mushy region, thereby reducing the turbulent kinetic energy values.

The kinematic viscosities for the two cases are presented in Figs. 6 and 7 to better illustrate the turbulence picture. As for the turbulent kinetic energy, the turbulent viscosity increases for lower values of $C$.

ii) Heat Transfer/Solidification Results

The temperature profile in the reservoir remained nearly unchanged, except for the region above the belt, where the metal is already mushy. These changes are shown in Figs. 8 and 9, for the smaller and bigger values of $C$. For the smaller $C$, the difference is best noticed near the nozzle entrance, 


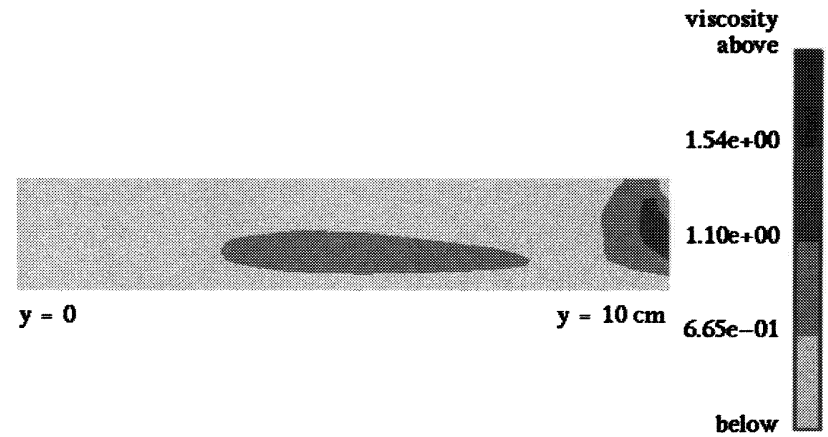

Fig. 6. Turbulent viscosity at the symmetry axis of the strip inside the reservoir - $C=1 \times 10^{5}$.

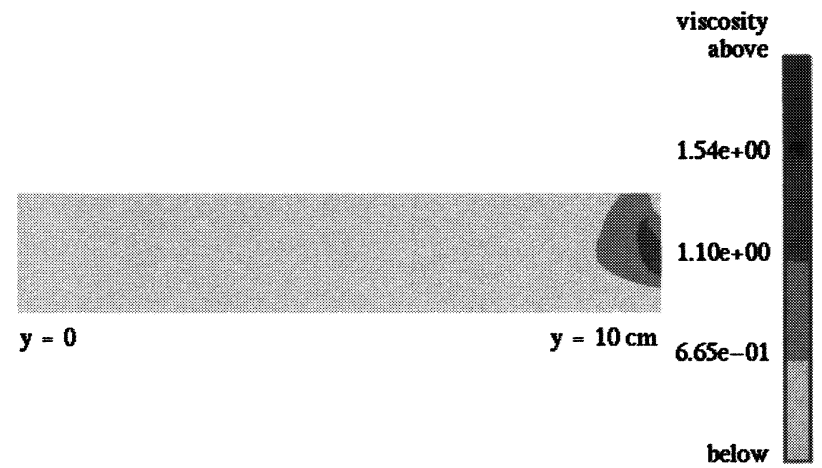

Fig. 7. Turbulent viscosity at the symmetry axis of the strip inside the reservoir - $C=1 \times 10^{9}$.

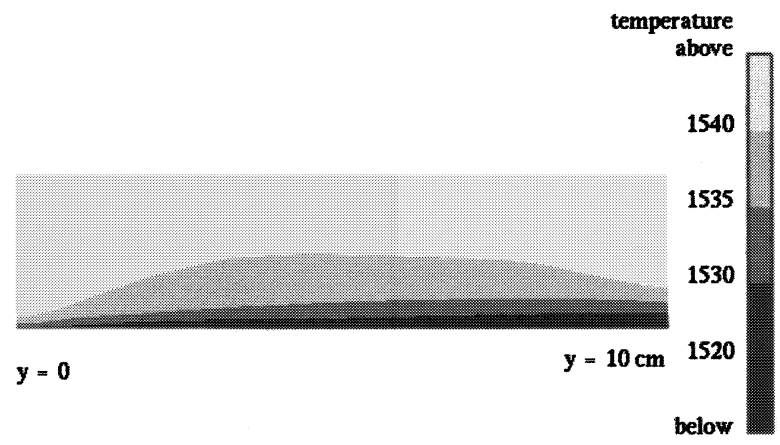

Fig. 8. Temperature profile at the symmetry axis of the strip inside the reservoir - $C=1 \times 10^{5}$.

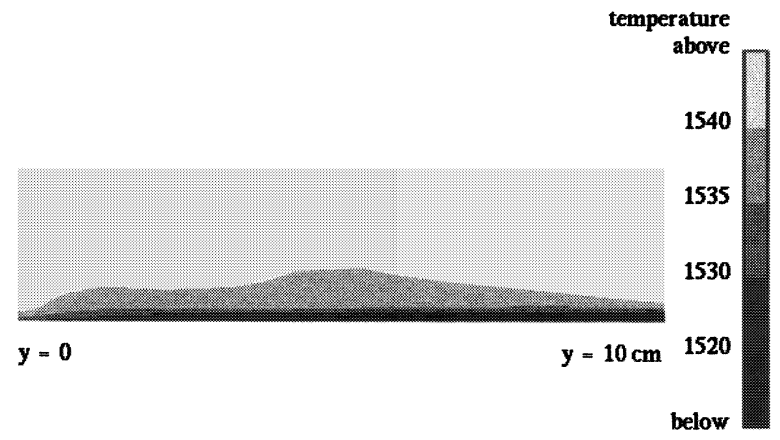

Fig. 9. Temperature profile at the symmetry axis of the strip inside the reservoir - $C=1 \times 10^{9}$.

where the mushy zone is enlarged and the reheating is not as strong as in the standard case. For the larger $C$, the modifications happened throughout the strip's length: the strip is hotter and the mushy zone is minimal at the exit gap.

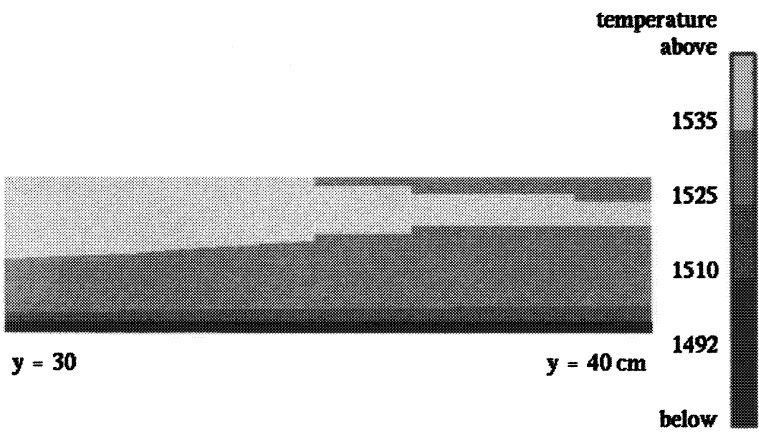

Fig. 10. Temperature profile at the symmetry axis of the strip in the final $10 \mathrm{~cm}$ of the computational domain $-C=1 \times 10^{5}$.

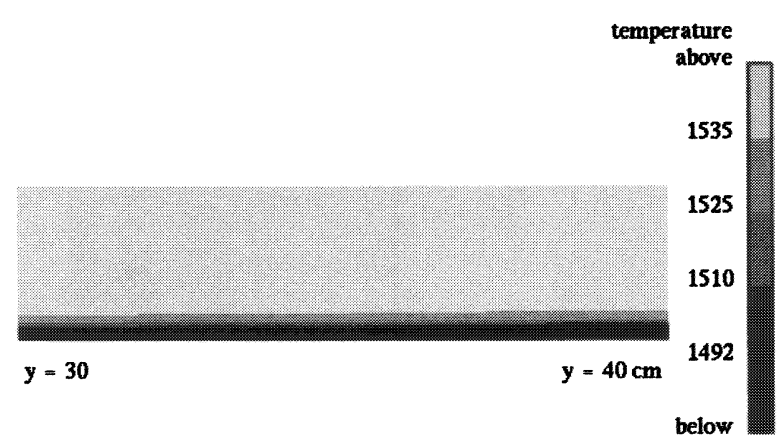

Fig. 11. Temperature profile at the symmetry axis of the strip in the final $10 \mathrm{~cm}$ of the computational domain $C=1 \times 10^{9}$.

Table 3. Average solid fraction at $y=40 \mathrm{~cm}$, for various values of $C$.

\begin{tabular}{cccc}
\hline $\mathbf{C}$ & $\mathbf{f}_{\mathbf{s}}$ (center) & $\mathbf{f}_{\mathbf{s}}$ (edge) & $\Delta$ \\
\hline $1 \times 10^{5}$ & 0.175 & 0.280 & 0.105 \\
$1 \times 10^{7}$ & 0.157 & 0.189 & 0.032 \\
$1 \times 10^{9}$ & 0.163 & 0.167 & 0.004 \\
\hline
\end{tabular}

Almost the whole strip is above $1540^{\circ} \mathrm{C}$ at this point, which will eventually retard the solidification.

At $40 \mathrm{~cm}$ from the meniscus, the temperature profiles are more representative of the mushy zone shape. Figures 10 and $\mathbf{1 1}$ show very clearly the effect of the Darcy coefficient. A small value for $C$ maximizes the mushy zone: here, almost no liquid is seen in the center of the strip after $40 \mathrm{~cm}$. On the contrary, an exaggeratedly high value for $C$ would considerably reduce the mushy region, confining it to a narrow region of approximately $1 \mathrm{~mm}$. To make up for this diminished mushy zone, the solidified shell is thicker: $1.3 \mathrm{~mm}$. This behavior would generate a "banded" form of solidification (i.e. a thin mushy zone sandwiched between the solid and liquid phases) which is in disagreement with our current experience and understanding of carbon steel solidification.

Table 3 shows that the divergence in solid fraction is still present, for the symmetry axis and for the edge of the strip, though it would be well reduced in a hypothetical "banded" solidification.

The simulations involving the Darcy coefficient $C$ illustrates the need for its accurate determination for precise temperature profiles and mushy zone morphology. As such, efforts to determine this parameter for the specific process 
should be encouraged.

\section{Effect of the Overall Heat Transfer Coefficient $h$}

Perhaps the most important boundary condition in a horizontal single-belt caster is the overall heat transfer coefficient $h$ applied to the metal interface. As discussed in Ref. 17), the thermal boundary conditions for near-net-shape casting processes vary markedly among the various processes. Values of $h$ up to $1 \mathrm{MW} / \mathrm{m}^{2} \cdot{ }^{\circ} \mathrm{C}$ have been report$\mathrm{ed}^{5)}$ for splat cooling processes, and as low as $700 \mathrm{~W} / \mathrm{m}^{2} \cdot{ }^{\circ} \mathrm{C}$ for slab casting in a twin-belt machine. ${ }^{16)}$ Therefore, each type of contact between the molten metal and the cooling substrate corresponds to distinct ranges of $h$.

Measuring the heat transfer coefficient in an actual horizontal belt machine is not an easy task. The moving belt restricts the positioning of thermocouples. Measurements of the thickness of the solidified shell and of microscopic parameters, such as the SDAS, are broadly used and values correlated with associated heat fluxes and heat transfer coefficients. In addition, laboratory scale trials have been attempted to determine $q$ and $h$ for the horizontal casting of aluminum and steel. ${ }^{16,20)}$ These earlier efforts, though relevant, failed to closely reproduce actual industrial conditions of wettability and relative velocity between the liquid metal and the substrate, since the laboratory trials were carried out using either a static system ${ }^{16)}$ or using speeds that were much lower than those intended for the single-belt caster configuration. ${ }^{20)}$

The purpose of this section is to determine how critical a precise determination of the thermal boundary conditions at the interface metal-cooling belt is during the first stages of solidification. For that, the initial approach of a constant value of $h$ throughout the length of the belt was replaced with two alternative strategies:

a) Stepwise variation of $h$, for the various regions of the caster.

A three-stage solidification process was considered for the first $40 \mathrm{~cm}$ of the belt. This assumption was based on the general knowledge that heat transfer conditions are nearly ideal during the first moments of casting and that the contact between the metal and the belt deteriorates as casting evolves, due to metal contraction and the formation of an air gap. The scheme used was as follows: for the part of the belt inside the reservoir, a maximum heat transfer coefficient of $10000 \mathrm{~W} / \mathrm{m}^{2} \cdot{ }^{\circ} \mathrm{C}$ was applied. Over the short distance of metal flow under the front wall, an intermediate value of $5000 \mathrm{~W} / \mathrm{m}^{2} \cdot{ }^{\circ} \mathrm{C}$ was considered and from the end of front wall to the end of the domain, an $h$ of 3000 $\mathrm{W} / \mathrm{m}^{2} \cdot{ }^{\circ} \mathrm{C}$ was adopted.

\section{i) Fluid Flow}

The most noticeable change in fluid flow in the reservoir using these heat fluxes is the "horizontalization" of the recirculating flow immediately above the cooling belt for the first half of the reservoir length, as shown in Figs. 12 and 13. The reason for this might have been the reduced effect of the drag force in the recirculating flow, due to a quicker formation of a solid and mushy zone. However, this alteration does not seem relevant, since most of the recirculating

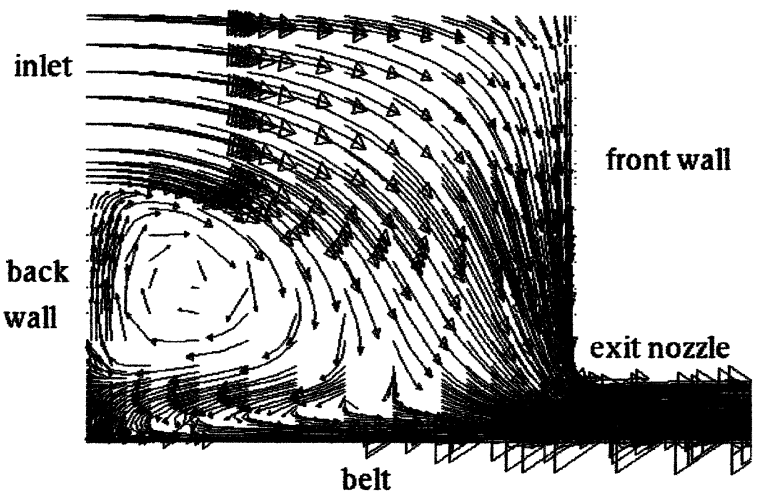

Fig. 12. Vertical cut along the symmetry axis - Uniform interfacial heat transfer coefficient $=5000 \mathrm{~W} / \mathrm{m}^{2} \cdot \mathrm{K}$.

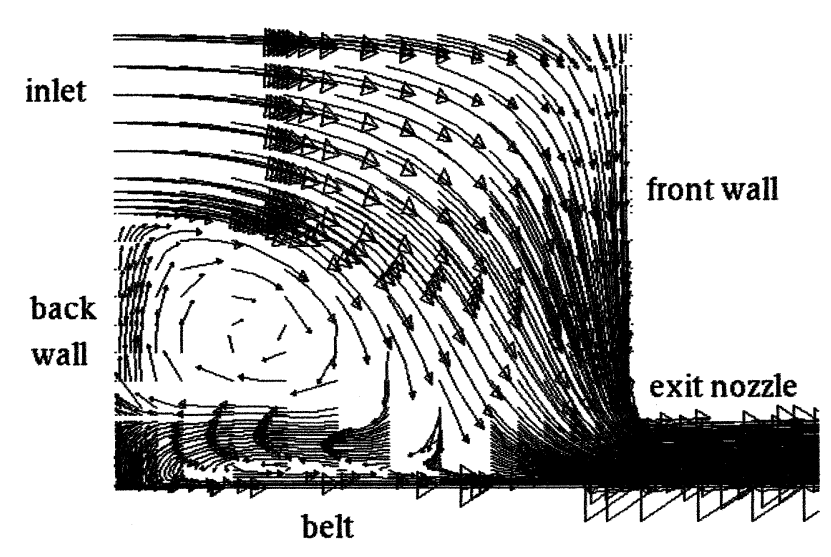

Fig. 13. Vertical cut along the symmetry axis - Stepwise variation of the heat transfer coefficient.

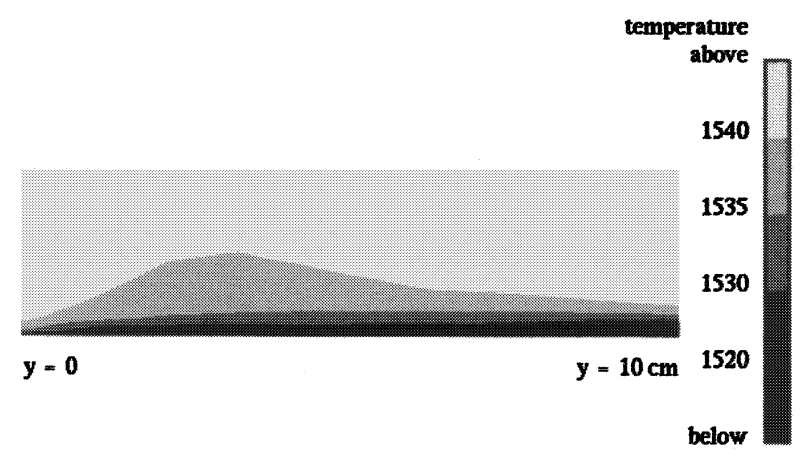

Fig. 14. Temperature profile at the symmetry axis of the strip inside the reservoir - Stepwise variation of the heat transfer coefficient.

flow is drawn down in the melt region next to the back wall, as in the standard condition.

In the other regions of the reservoir, the flow patterns are nearly identical to the standard ones. The turbulence profiles also indicate that the effect is limited to the region immediately above the cooling belt.

ii) Heat Transfer/Solidification Results

The doubled heat transfer coefficient in the reservoir zone is responsible for the formation of a thin solidified shell within the reservoir. Another interesting finding is that the temperature profile is suddenly changed at approximately $3 \mathrm{~cm}$ from the meniscus ( $y=3 \mathrm{~cm}$, Fig. 14), switching from cooling to reheating. This reversion may not be credit- 


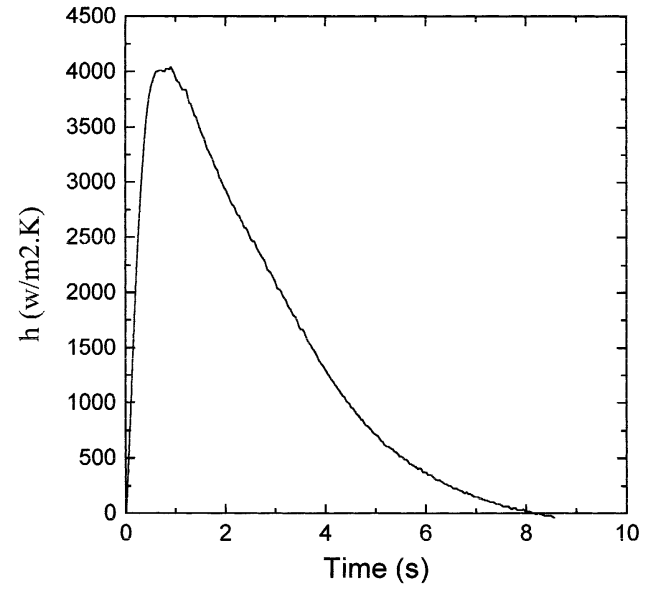

Fig. 15. Calculated interfacial heat transfer coefficients for the casting of steel in the experimental apparatus.

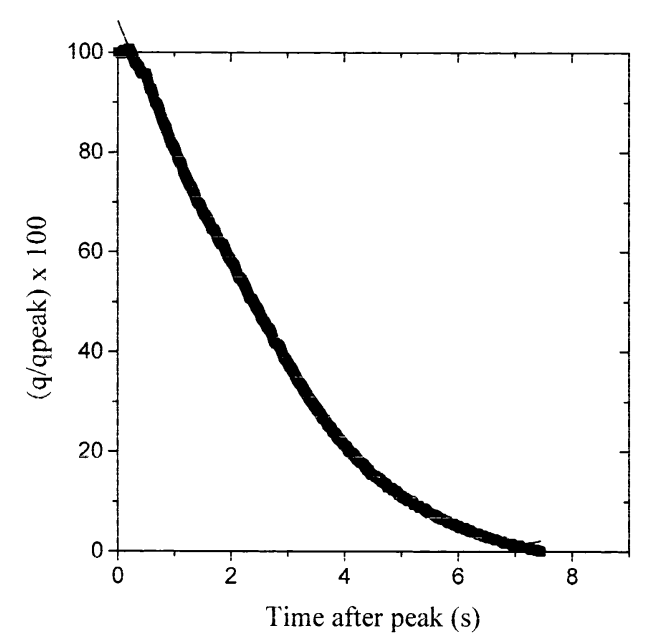

Fig. 16. Variation in normalized interfacial heat fluxes versus contact times following the peak in interfacial heat flux, for the casting of steel in the experimental apparatus.

ed to the effect of the incoming flow, but rather to the changes in the flow pattern mentioned above. The premature solidification contributes to damp turbulence right above the belt, and the liquid region above the solid/mushy zone has its cooling retarded.

b) Transient evolution of $h$, as given by the experiments carried out with the strip casting simulator. ${ }^{17)}$

Figure 15 shows the evolution of the heat transfer coefficient in a typical experiment carried out in an apparatus where the substrate is moving at speeds in the same order as those intended for the single-belt caster $(\sim 1 \mathrm{~m} / \mathrm{s})$. To input the variation of $h$ into the code in a simplified manner, two stages were considered: the first is a linear variation of $h$ from time zero up to the peak (going from zero at $t=0$ to maximum at $t_{\text {peak }}$ ) and the second is the decay of $h$ according to the following formula for the evolution of the heat flux:

$$
\frac{q}{q_{\mathrm{p}}}=1.04-0.275 t+0.00144 t^{2}+5 \times 10^{-4} t^{3}
$$

Figure 16 shows the excellent fit given by this correlation to the experimental data obtained in the apparatus. For $t=0$ and very small times, a restriction was imposed on the
Table 4. Average solid fractions and solid shell thicknesses at $1.5 \mathrm{~m}$ from the meniscus (entry wall), for different schemes of $h$.

\begin{tabular}{cccc}
\hline Description & $\mathrm{f}_{\mathrm{s}}$ (center) & $\mathrm{f}_{\mathrm{s}}($ edge $)$ & $\mathrm{X}(\mathrm{mm})$ \\
\hline Constant $\mathrm{h}$ & 0.393 & 0.436 & 2.60 \\
Stepwise variation & 0.335 & 0.386 & 2.20 \\
Transient evolution & 0.278 & 0.342 & 1.80 \\
\hline
\end{tabular}

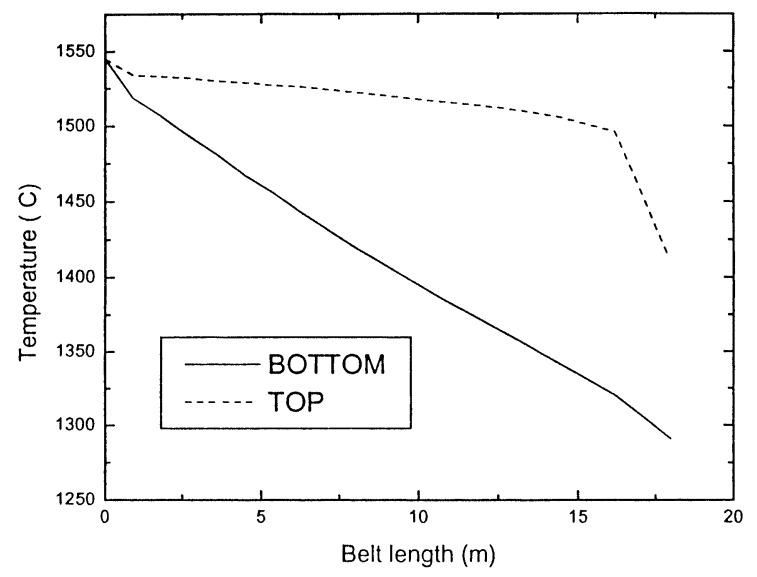

Fig. 17. Calculated temperature profile for the bottom and top of a 10 -mm strip, considering a uniform $h=1000 \mathrm{~W} / \mathrm{m}^{2} \cdot \mathrm{K}$ on the bottom and radiation on the top.

Table 5. Total solidification time, for various values of $h$.

\begin{tabular}{cc}
\hline Value of $h\left(\mathrm{~W} / \mathrm{m}^{2} . \mathrm{K}\right)$ & Solidification time $(\mathrm{s})$ \\
\hline 5000 & 7.5 \\
3000 & 9.8 \\
1000 & 17.5 \\
\hline
\end{tabular}

code, to avoid a value of $h$ higher than the peak. The most important advantage in using this data is that they were obtained under conditions fairly similar to actual ones. One may find different values for different belt substrates and coatings, but the shape of the curve is believed to remain approximately the same.

To summarize the analysis, Table 4 provides a comparison between the average solid fractions and thicknesses of the solid shell at $1.5 \mathrm{~m}$ from the meniscus, for the three cases considered for $h$ : uniform value, stepwise variation and transient evolution. The results stress the importance of an accurate determination of the way $h$ values change with time.

\section{- Estimation of the Length of the Cooling Belt}

A two-dimensional heat conduction model was employed in order to estimate the necessary length of the cooling belt, for different averaged values of $h$. The dimensions of the strip were the same, as well as the thermophysical properties and the inlet superheat. Heat was lost by radiation and convection on the top surface and an overall heat transfer coefficient was applied for the spray cooling. As the cooling conditions on the bottom are much stronger than on the top, a nearly unidirectional cooling is expected for the relatively thin strip $(10 \mathrm{~mm})$, as shown in Fig. 17. This figure portrays the predicted temperature evolution on the bottom 
and on the top of the strip, for an average $h$ of $1000 \mathrm{~W} / \mathrm{m}^{2} \cdot \mathrm{K}$. Indeed, Kim et al. ${ }^{21)}$ found similar results for planar flow casting of 3-mm thick strips. In this system, for very thin strips the heat transfer is limited by the interfacial resistance, given the small thickness and the relatively high thermal conductivity of the metal.

Table 5 shows the predicted solidification times, considering three different averaged values for $h$. The predictions are in reasonable agreement with the values suggested by Spitzer and Schwerdtfeger. ${ }^{4)}$ In their paper, they present a graph of solidification time versus strand thickness. For a 10 -mm strip, they predict approximately $15 \mathrm{~m}$ for the belt.

\section{Conclusions}

- A mathematical model was developed for the prediction of fluid flow/ heat transfer and solidification phenomena occurring in a proposed configuration for a single-belt caster. The model included turbulent flow, according to a $\kappa-\varepsilon$ model and also flow through a porous medium;

- A study was conducted on the influence of the most important inputs of the model on the predictions of heat transfer/solidification phenomena. These parameters were found to be: the interfacial heat transfer coefficient $h$ and the morphology constant $C$, both of which should be empirically determined;

- The simulations showed that the precise determination of the morphology constant $C$ is crucial if the size and position of the mushy zone is to be determined. A relatively high value of $C$ will dampen velocities in the mushy zone more strongly. As a result, a "banded" solidification profile is predicted, with a relatively thick solid shell, a very thin mushy zone and a large region of liquid above. On the other hand, a relatively low value of $C$ will yield a wider mushy zone and relatively small liquid and solid phases. A coupled micro and macroscopic model would also be useful to elucidate the solidification mechanism and to provide a more accurate prediction of the morphology of the mushy zone;

- The most important thermal boundary condition of the model is the interfacial heat flux or interfacial heat transfer coefficient. The interfacial resistance is the limiting step for heat transfer, for the very thin strips intended to be cast in this machine. For the various thin-strip-casting processes in development, different kinds of metal-substrate interfacial contact occur, which makes very important a more precise determination of the heat transfer coefficient evolution. For an industrial single-belt caster process, this information is important in the designing of the caster, since the belt length will ultimately be calculated according to the interfacial thermal resistance.

\section{REFERENCES}

1) A. W. Cramb: Proc. of the Int. Symp. on Near Net Shape Casting in the Minimills, 34th Annual Conference of Metallurgists, CIM, Monereal, (1995), 355.

2) H. Murakami: Ph. D. Thesis, Dep. of Mining and Metallurgical Engineering, McGill University, (1993), 5.

3) A. W. Cramb: Proc. of Melt Spinning, Strip Casting and Slab Casting, TMS, Warrendale, PA, (1996), 1.

4) K. H. Spitzer and K. Schwerdtfeger: Iron Steelmaker, 22 (1995), 47.

5) G. X. Wang and E. F. Matthys: Proc. of Melt Spinning, Strip Casting and Slab Casting, TMS, Warrendale, PA, (1996), 205.

6) P. G. Q. Netto and R. I. L. Guthrie: Int. Journ. of Heat and Mass Transfer, 43 (2000), 21.

7) S. V. Patankar: Numerical Heat Transfer and Fluid Flow, Hemisphere Publishing Corp., New York, (1980), 15.

8) W. D. Bennon and F. P. Incropera: Int. Journ. of Heat and Mass Transfer, 30 (1987), No. 10, 2161.

9) A. D. Brent, V. R. Voller and K. J. Reid: Numer. Heat Transfer, 13 (1988), 297.

10) Shyy, Y. Pang, G. B. Hunter, D. Y. Wei and M. H. Chen: Int. Journ. of Heat and Mass Transfer, 35 (1992), No 5, 1229.

11) B. E. Launder and B. I. Sharma: Let. in Heat and Mass Transfer, 1 (1974), 131.

12) M. Reza Aboutalebi, M. Hasan and R. I. L. Guthrie: Metall. Mater. Trans. B, 26B (1995), 731.

13) S. H. Seyedein: Ph. D. Thesis, McGill University, Dep. of Mining and Metallurgical Engineering, (1997), 1.

14) R. P. Tavares: Ph. D. Thesis, McGill University, Dep. of Mining and Metallurgical Engineering, (1997), 1.

15) I. F. McDonald, M. S. El-Sayed, K. Mow and F. A. L. Dullien: Ind. Eng. Chem. Fundam., 18 (1979), 199.

16) B. Farouk, D. Apelian and Y. G. Kim: Metall. Trans. B, 23B (1992), 477.

17) P. G. Q. Netto: Ph. D. Thesis, McGill University, Dep. of Mining and Metallurgical Engineering, (1998), 1.

18) S. Minakawa, I. V. Samarasekera and F. Weinberg: Metall. Trans. B, 16B (1987), 245.

19) C. Jefferies: Ph. D. Thesis, McGill University, Dep. of Mining and Metallurgical Engineering, (1995), 1.

20) A. Couture, R. Angers, M. R. Krishnadev, E. Es-Sadiqi and J. Masounave: Can. Metall. Q., 31, (1992), No. 1, 63.

21) Y. Kim, B. Farouk and J. Keverian: J. Eng. Ind. (Trans. ASME), 113 (1991), 53. 